\title{
A Functional Polymorphism of the MAOA Gene Modulates Spontaneous Brain Activity in Pons
}

\author{
Hui Lei, ${ }^{1}$ Xiaocui Zhang, ${ }^{1}$ Xin Di, ${ }^{2}$ Hengyi Rao, ${ }^{3,4}$ Qingsen Ming, ${ }^{1}$ Jibiao Zhang, \\ Xiao Guo, ${ }^{1}$ Yali Jiang, ${ }^{1}$ Yidian Gao, ${ }^{1}$ Jinyao $Y i,{ }^{1}$ Xiongzhao Zhu, ${ }^{1}$ and Shuqiao Yao ${ }^{1}$ \\ ${ }^{1}$ The Medical Psychological Institute of the Second Xiangya Hospital, Central South University, Changsha, Hunan 410011, China \\ ${ }^{2}$ Department of Biomedical Engineering, New Jersey Institute of Technology, Newark, NJ 07102, USA \\ ${ }^{3}$ Center for Functional Neuroimaging, Department of Neurology, University of Pennsylvania, Philadelphia, PA 19104, USA \\ ${ }^{4}$ Department of Psychology, Sun Yat-Sen University, Guangzhou, Guangdong 510275, China
}

Correspondence should be addressed to Shuqiao Yao; shuqiaoyao@163.com

Received 12 March 2014; Revised 13 May 2014; Accepted 14 May 2014; Published 25 May 2014

Academic Editor: Yong He

Copyright (c) 2014 Hui Lei et al. This is an open access article distributed under the Creative Commons Attribution License, which permits unrestricted use, distribution, and reproduction in any medium, provided the original work is properly cited.

\begin{abstract}
Objective. To investigate the effects of a functional polymorphism of the monoamine oxidase A (MAOA) gene on spontaneous brain activity in healthy male adolescents. Methods. Thirty-one healthy male adolescents with the low-activity MAOA genotype (MAOA-L) and 25 healthy male adolescents with the high-activity MAOA genotype (MAOA-H) completed the 11-item Barratt Impulsiveness Scale (BIS-11) questionnaire and were subjected to resting-state functional magnetic resonance imaging (rs-fMRI) scans. The amplitude of low-frequency fluctuation (ALFF) of the blood oxygen level-dependent (BOLD) signal was calculated using REST software. ALFF data were related to BIS scores and compared between genotype groups. Results. Compared with the MAOA$\mathrm{H}$ group, the MAOA-L group showed significantly lower ALFFs in the pons. There was a significant correlation between the BIS scores and the ALFF values in the pons for MAOA-L group, but not for the MAOA-H group. Further regression analysis showed a significant genotype by ALFF values interaction effect on BIS scores. Conclusions. Lower spontaneous brain activity in the pons of the MAOA-L male adolescents may provide a neural mechanism by which boys with the MAOA-L genotype confers risk for impulsivity and aggression.
\end{abstract}

\section{Introduction}

Monoamine oxidase (MAO) is a mitochondrial enzyme that was involved in degradation of neurotransmitters, including norepinephrine (NE), serotonin (5-HT), and dopamine [1]. There are two monoamine oxidase isozymes with distinct substrate specificities: MAOA and MAOB. MAOA provides the major enzymatic clearance of 5-HT and NE during brain development [1]. The MAOA-encoding gene (Xp11.4-Xp11.3) presents a well-characterized variable number tandem repeat (VNTR) functional polymorphism in the promoter region, with different length variants that influence protein transcription, and hence enzymatic activity, selectively $[2,3]$. Enzyme expression is relatively higher in carriers of 3.5 or 4 repeats (MAOA-H allele) and lower in carriers of 2, 3, or 5 repeats (MAOA-L allele) [2]. Converging evidence indicates that this functional polymorphism has a strong influence on serotonergic function in vitro and in vivo [2-4], with the high activity allele showing lower serotonergic responsivity [4].

Previous behavioral researches have indicated an important role for MAOA in human behavior and physiology. Caspi and colleagues found that males carrying MAOA-L alleles who experienced early-life adversity had a heightened risk of developing conduct disorder or antisocial personality and of exhibiting violent and antisocial behavior [5]. Besides, Samochowiec et al. found that the MAOA-L allele has been associated with antisocial behavior in male alcoholdependent patients [6]. Huang et al. found a significant correlation of the MAOA-H allele with lower impulsivity in adult males who report early childhood abuse, further supported an association of MAOA-L allele and impulsivity in males [7]. Subsequent studies, including one meta-analysis 
[8], have replicated the findings independently [9-11]. Conversely, the MAOA-H allele has been associated with an even greater propensity for antisocial behavior and impulsivity than the MAOA-L allele in males who experience early stress $[12,13]$. Moreover, the MAOA-H allele has been associated with impulsive personality traits in normal male subjects [4]. Animal studies indicated that MAOA had an important effect on aggressive behavior. Aggression is increased in the male MAOA knockout mice [14] and monkeys with reared experience [15]. MAOA inhibition during brain development induced pathological aggression in mice [16].

These findings suggest that genetically driven variations in MAOA activity significantly influence impulsivity and aggression.

Using noninvasive neuroimaging techniques, including both positron emission tomography (PET) and functional magnetic resonance imaging (fMRI), previous studies have examined the effect of this polymorphism on brain function. Previous fMRI studies have demonstrated that individuals carrying MAOA-H alleles showed increased cingulate activation during conflict resolution [17] and increased orbitofrontal cortex activation during performing motor inhibition and working memory tasks [18, 19]. MeyerLindenberg et al. also reported that the MAOA polymorphism had a profound impact on the corticolimbic circuitry involved in emotional memory [20]. Structural MRI studies employing voxel-based morphometry (VBM) also produced conflicting findings with respect to the effect of MAOA genotypes on amygdala volume [20-22]. However, a positron emission tomography (PET) study found no differences in the glucose metabolism between MAOA-L and MAOA-H groups [23]. Currently, the neurobiological mechanisms underlying the effects of the MAOA polymorphism on impulsiveness are still unclear.

During the last decade, studies of spontaneous brain activity at resting states in normal individuals as well as patients with brain diseases have attracted enormous research interests. Biswal and colleagues firstly demonstrated in human subjects that spontaneous low-frequency $(0.01 \sim$ $0.08 \mathrm{~Hz}$ ) brain fluctuations measured by resting-state fMRI are physiologically meaningful [24]. The amplitude of lowfrequency fluctuation (ALFF) of BOLD signals was developed by Zang and colleagues to provide another measurement of regional neural function during a resting-state [25]. Previous studies have suggested that ALFF is physiologically meaningful and reflective of regional spontaneous neuronal activity [25-27]. To date, ALFF analysis has been applied widely to the studies of different brain disorders, including epilepsy [28], schizophrenia [29, 30], major depressive disorder [31, 32], drug addiction [33], posttraumatic stress disorder [34], attention deficit hyperactivity disorder [25], and multiple sclerosis [35]. However, it remains unknown whether restingstate spontaneous brain activity would differ as a function of MAOA genetic variation. The purpose of this study is answering this question by comparing the ALFF in matched MAOA-L and MAOA-H male adolescents. Furthermore, ALFF measurements were correlated with impulsivity scores as measured by the Barratt Impulsiveness Scale (BIS) in both MAOA-L and MAOA-H genotypes, in order to examine the neural bases underlying the effects of the MAOA polymorphism on impulsive behavior.

\section{Materials and Methods}

2.1. Participants. A total of 60 healthy male adolescents were recruited from a local middle school. All of them were Han Chinese. Only male adolescents were included because the MAOA VNTR polymorphism maps to an X-chromosome region suspected to escape the normal X-chromosome inactivation in females [36], making homozygous females not comparable to hemizygous males in terms of enzymatic activity. The inclusion criteria were an ability to give voluntary informed consent, an absence of concurrent neurological or psychiatric disorders, no history of head trauma, alcohol or drug abuse, no history of psychiatric illness or substance abuse on the basis of a SCID I assessment for DSM-IV criteria (1994), and no history of medical treatments relevant to cerebral blood flow and metabolism. The study was approved by the Ethics Committee of the Second Xiangya Hospital at Central South University in China, and all participants gave written informed consent to participate.

2.2. Neuropsychological Evaluation. The Italian version of the 11-item Barratt's Impulsivity Scale (BIS-11) was administered to obtain neuropsychological profiles of the subjects' impulsivity [37].

2.3. Genotyping. Samples of DNA were obtained by cheek swabbing. Polymerase chain reactions (PCRs) were performed in $25 \mu \mathrm{L}$ reaction volumes containing $1 \mu \mathrm{L}$ of DNA, GoTaq Green Master Mix (Promega Company, USA), $12.5 \mu \mathrm{L}$

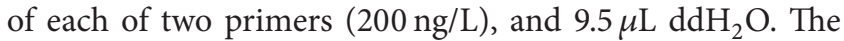
amplification protocol was as follows: $94^{\circ} \mathrm{C}$ for $3 \mathrm{~min}, 35$ cycles of $95^{\circ} \mathrm{C}$ for $30 \mathrm{~s}, 58^{\circ} \mathrm{C}$ for $30 \mathrm{~s}$, and $72^{\circ} \mathrm{C}$ for $45 \mathrm{~s}$ in a Gene Amp PCR system 2400 (Applied Biosystem, CA). PCR products were separated and electrophoresed on a $1.8 \%$ agarose gel and stained with Du Red (Biosharp, USA). They were then viewed under UV transillumination, and sizes were determined by comparison with a 50 bp DNA sequencing ladder. The MAOA-VNTR (MAOA promoter region polymorphism) was genotyped according to previously described methods [2]. Alleles with 2, 3, or 5 repeats were categorized as "low" activity, while those with 3.5 or 4 repeats were categorized as "high" activity.

2.4. Data Acquisition. All fMRI scans were obtained with a Philips Achieva 3-T scanner. The participants were instructed to keep their eyes closed and refrain from initiating goaldirected, attention-demanding activity during scanning. Foam pads were used to reduce head movements, and fitted ear plugs were used to reduce scanner noise. Resting-state fMRI scans were performed with an echo planar imaging sequence. Scan parameters were as follows: repetition time $=$ $2000 \mathrm{~ms}$; echo time $=30 \mathrm{~ms}$; flip angle $=90^{\circ}$, matrix $=64 \times 64$; field of view $=240 \mathrm{~mm}$; slice thickness $=4 \mathrm{~mm}$; and slice gap $=$ $0 \mathrm{~mm}$. Each brain volume contained 36 axial slices, and each functional run contained 206 volumes. Before the functional 
scan, high-resolution $\left(1 \times 1 \times 1 \mathrm{~mm}^{3}\right)$ anatomical images of 180 contiguous slices were obtained with a 3D MPRAGE sequence (repetition time $=8.5 \mathrm{~ms}$, echo time $=3.7 \mathrm{~ms}$, and flip angle $=8^{\circ}$ ).

2.5. Data Preprocessing. Image preprocessing and statistical analyses were performed with Statistical Parametric Mapping (SPM8, http://www.fil.ion.ucl.ac.uk/spm/). The first six volumes of the functional images were discarded for magnetization equilibrium and participants' adaptation to scanning noise. For each participant, functional images were motion corrected using the realignment function. Data from the subjects whose head motions exceeded $2 \mathrm{~mm}$ in the $x, y$, or $z$ plane or whose rotation exceeded $2^{\circ}$ during scanning were excluded.

Each subject's anatomical images were segmented using the new segment function, and deformation field maps were obtained. The deformation field maps were applied to all functional images to normalize them into the standard Montreal Neurological Institute (MNI) space with a resampling voxel size of $3 \times 3 \times 3 \mathrm{~mm}^{3}$. Segmented anatomical images (unmodulated) were used to define white matter (WM) and cerebrospinal fluid (CSF) masks by thresholding the density images at a value of 0.99 . For the time series of each voxel, the first eigenvector of the time series in the WM mask and the first eigenvector of the times series in the CSF mask, together with 24 motion parameters of Friston's model [38], were regressed out using linear regression. Finally, the time series of each voxel was filtered temporally using a bandpass filter $(0.01-0.1 \mathrm{~Hz})$ to reduce the effects of low-frequency drifts and of physiological high frequency respiratory and cardiac noises.

2.6. ALFF Analysis. The REST software package (REST, http://resting-fmri.sourceforge.net) was used to calculate ALFF values with a voxel-based approach. The filtered time series for each voxel were transformed to the frequency domain with a Fast Fourier Transform function, yielding a power spectrum. The square root of the power spectrum was calculated and then averaged across $0.01-0.08 \mathrm{~Hz}$ at each voxel. This averaged square root was taken as the ALFF [25]. To reduce global effects of variability across participants, the ALFF of each voxel was divided by the global mean ALFF value within the whole-brain mask obtained previously, and a standardized ALFF map of the whole brain was obtained. The ALFF maps were then smoothed spatially with an $8 \mathrm{~mm}$, full width at half maximum (FWHM) Gaussian filter.

2.7. Analyses of Clinical Variables in relation to ALFF Data. To investigate the relationship between altered ALFF and impulsivity scores, the average ALFF values of all voxels were extracted separately, and then Pearson's correlation coefficients for ALFF values versus BIS-11 total scores were computed for each group separately.

2.8. Statistical Analysis. Two-sample $t$-tests were applied to assess demographic and clinical data differences between MAOA genotype groups in SPSS 11.6 software (SPSS Inc.,
TABLE 1: Demographic information of subjects.

\begin{tabular}{lccc}
\hline Characteristic & $\begin{array}{c}\text { MAOA-H } \\
(N=25)\end{array}$ & $\begin{array}{c}\text { MAOA-L } \\
(N=31)\end{array}$ & $P$ value \\
\hline Age, years: mean (s.d.) & $15.92(0.81)$ & $15.55(0.81)$ & 0.09 \\
Education, year: mean (s.d.) & $10.56(0.51)$ & $10.52(0.57)$ & 0.77 \\
BIS-11 score: mean (s.d.) & $69.16(7.17)$ & $66.71(6.05)$ & 0.17 \\
\hline
\end{tabular}

Abbreviations: MAOA-H = high-activity MAOA genotype group; MAOA-L = low-activity MAOA genotype group;

BIS-11 = Italian version of the 11-item Barratt's Impulsivity Scale; s.d. = standard deviation.

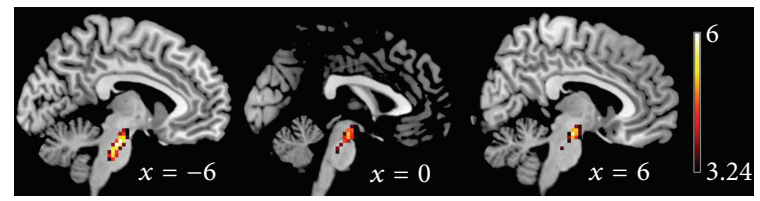

FIGURE 1: ALFF values using two-sample $t$-tests during resting state. Regions showing decreased ALFF values in male adolescents with MAOA-L compared to adolescents with MAOA-H were at the threshold $t>3.24$, with correction for multiple comparisons applied at $P<.05$ (cluster-corrected with family wise error). Color bar indicates the $T$ score.

Chicago, IL). To investigate ALFF differences between the two groups, two-sample $t$-tests were performed on the individual normalized ALFF maps in SPM8 software with age and educational level as nuisance covariates. The resulting statistical map was set at a combined threshold of $P<.001$ and a minimum cluster size of 50 , which resulted in a clustercorrected family-wise error $P<.05$.

\section{Results}

3.1. Genotyping and Participant Exclusions. The original cohort of 60 boys included 34 with the low-activity allele (57\%) and 26 with the high-activity allele (43\%). One highactivity allele and three low-activity allele subjects were excluded due to excessive head motion or rotation during scanning, leaving 31 low-activity allele and 25 high-activity allele subjects in the final analysis.

3.2. Demographic and Clinical Comparisons. The demographic and clinical data obtained for the two MAOA genotype groups are summarized in Table 1 . There were no differences between the two genotype groups in terms of age, years of education, or BIS scores (all $P>0.05$ ).

3.3. ALFF. Compared with MAOA-H carriers, adolescents carrying the MAOA-L allele showed significantly reduced ALFF values in the pons region of the brainstem (Table 2, Figure 1).

3.4. Behavioral Correlation. Although there were no differences in BIS scores between the MAOA genotype groups, only the MAOA-L group showed a significant correlation between the BIS score and the ALFF values in the pons 
TABLE 2: Regions that showed significant differences in ALFF values between the high-activity and low-activity MAOA genotype groups in resting-state.

\begin{tabular}{|c|c|c|c|c|c|c|c|}
\hline \multirow{2}{*}{ Bran region } & \multirow{2}{*}{ Side } & \multicolumn{3}{|c|}{ Peak MNI coordinates (mm) } & \multirow{2}{*}{ Cluster size } & \multirow{2}{*}{$Z$ score } & \multirow{2}{*}{$P$ value ${ }^{*}$} \\
\hline & & $X$ & $Y$ & $Z$ & & & \\
\hline \multicolumn{8}{|c|}{ MAOA-L $<$ MAOA-H } \\
\hline \multirow{3}{*}{ Pons } & $\mathrm{L}$ & -6 & -19 & -23 & \multirow{3}{*}{130} & 4.38 & \multirow{3}{*}{$<0.001$} \\
\hline & $\mathrm{R}$ & 6 & -16 & -17 & & 4.12 & \\
\hline & $\mathrm{L}$ & -6 & -25 & -32 & & 4.01 & \\
\hline
\end{tabular}

Abbreviations: ALFF= amplitude of low-frequency fluctuations; MAOA-H = high-activity MAOA genotype group; MAOA-L = low-activity MAOA genotype group; L= left; $\mathrm{R}=$ right; $\mathrm{MNI}=$ Montreal Neurological Institute.

${ }^{*}$ Cluster-corrected with family-wise errors.
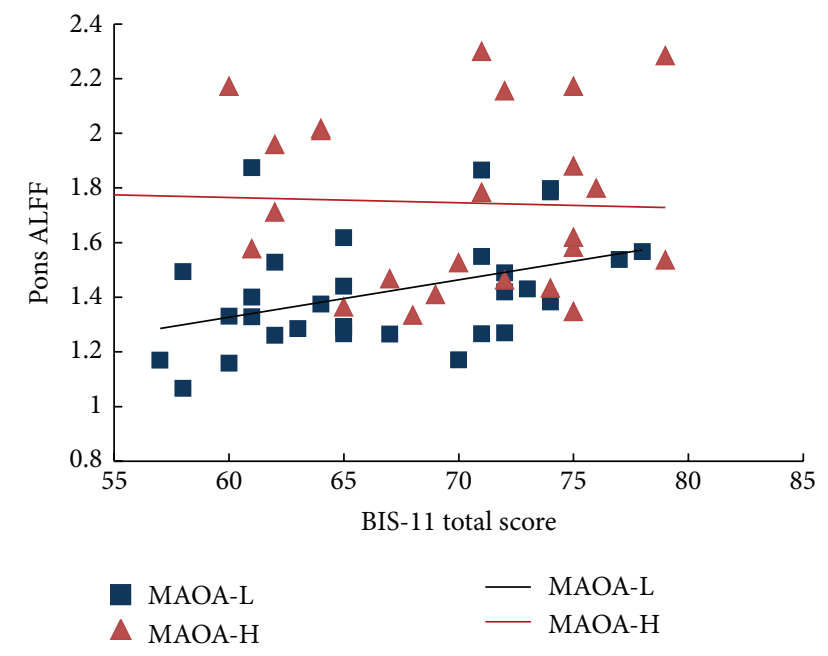

FIgURE 2: Correlations between BIS-11 total scores and pons ALFF values in MAOA-L and MAOA-H genotype groups.

( $r=0.398, P=.02$, Figure 2), MAOA-H group showed no such correlation $(r=-0.044, P=.833$, Figure 2). Further regression analysis showed a significant genotype by ALFF values interaction effect on BIS scores $(\beta=0.803, P<.05)$.

\section{Discussion}

To our knowledge, the present study is the first to reveal the influence of MAOA genotype on brain regional spontaneous activity in male adolescents. We found that male adolescents with the MAOA-L genotype had significantly lower ALFF values in the pons than MAOA-H counterparts. Given that ALFF changes are suggestive of regional spontaneous neuronal activity [25], this difference is consistent with the possibility that boys carrying the MAOA-L may have a neural impairment in the pons and points to differential effects on spontaneous brain activity between the two MAOA genotypes. The allelic distribution observed in our sample population was similar to those reported previously in Asian populations $[39,40]$, and the approximate inverse of ratios reported for Caucasian populations (60-70\% high activity allele and $30-40 \%$ low activity allele) $[5,22]$.
BIS-11 scores did not differ significantly between the groups, likely due to the small sample size in our study. Interestingly, however, we found that ALFF values in the pons correlated with BIS scores selectively in boys with a MAOA$\mathrm{L}$ genotype but not in MAOA-H group, and a significant genotype by ALFF interactive effect on impulsiveness. These results suggest that MAOA genotype may modulate the association of impulsiveness and ALFF in pons, and the association was only shown in MAOA-L group.

The pons, a major component of the brainstem, serves mainly as a relay center between the spinal cord, cerebellum, and cerebral cortices and neural centers that control respiration, heartbeat, reflexes, sleep, equilibrium, and auditory and visual functions $[41,42]$. Within the pons region, the locus coeruleus and raphe nuclei are important cranial nerve nuclei and are the principal sites for brain synthesis of NE and 5-HT, respectively. Using MAOA cDNA and oligonucleotide probes, Jahng et al. found that the pons (especially the locus coeruleus) is the region with the highest density of MAOA mRNA in the rat brain [43]. The MAOA gene polymorphism regulates the metabolism of NE and 5-HT [1]; animal and human studies indicate that the serotonin system has an important effect on aggressive and impulsive behavior [44, 45]. Therefore, it is possible that $M A O A$ genotype-dependent modulation of 5-HT and NE neurotransmission underlie the $M A O A$ gene effect to impulsiveness. Besides, it is possible that the influence of $M A O A$ on spontaneous neuronal activity in pons is most likely mediated through its effects on serotonin and dopamine.

Previous research has implicated the role of pons in aggression. For example, pontine lesions have been associated with aggressive behaviors in animals $[39,40]$. A human PET study showed reduced brain MAOA activity in the pons of participants who had high trait aggression compared with that in nonaggressive participants [41]. Indeed, several previous reports have pointed to the MAOA-L allele as a "risk" variant (allele) for impulsivity and aggression. Since Caspi and colleagues discovered that males carrying MAOA-L alleles who experienced early-life adversity were significantly likely to evince conduct disorder, an antisocial personality, and exhibit violent, antisocial behavior [5], a number of investigators have replicated the similar founding [9-11]. In addition, Huang et al. found that people with MAOA-L allele displayed more aggression and impulsive tendencies experienced abuse relative to their peers with MAOA-H allele 
[7]. Our findings of low ALFF values in the pons of MAOA-L allele carriers, relative to those of MAOA-H allele carriers and selective correlation of BIS-11 scores with pons ALFF values in the MAOA-L group fit well the possibility that the MAOA$\mathrm{L}$ allele may indeed be such a risk variant.

Our findings contrast with the results from a recent PET study showing no significant differences in resting brain glucose metabolism between the MAOA-L and MAOA$\mathrm{H}$ genotypes [23]. However, previous fMRI studies have revealed significant effects of $M A O A$ polymorphisms on brain activation in multiple corticolimbic regions, including anterior cingular cortex, orbitofrontal cortex, and amygdala [17-20]. The inconsistent findings might be due to the different methodologies used and differences in the participants' characteristics, especially with respect to age composition.

There are some limitations of the current study that warrant consideration. First, the sample size is really small. We cannot rule out the possibility of false positive findings and the possibility of the gene effect on other brain regions. Second, only males were included in our study. We cannot determine whether the results are specific to males or also relate to females. Future studies should include both males and females with a larger sample size. In addition, the $M A O A$ promoter polymorphism is likely not the only $M A O A$ variant that has an effect on impulsiveness or brain function. We cannot rule out the potential interactive contribution of genotype-phenotype and gene-environment to impulsiveness or brain function.

\section{Conclusions}

In summary, in the present study, we obtained evidence showing that a well-characterized functional polymorphism in the MAOA gene modulates resting-state spontaneous brain activity in the pons in healthy male adolescents. Lower $\backslash$ spontaneous brain activity in the pons of the MAOA-L male adolescents may provide a neural mechanism by which boys with the MAOA-L genotype confers risk for impulsivity and aggression.

\section{Conflict of Interests}

The authors declare that there is no conflict of interests in this paper with any trademark or software mentioned.

\section{Authors' Contribution}

Hui Lei and Xiaocui Zhang made equal contributions to this study.

\section{Acknowledgments}

This work was supported by a grant from the Ph.D. Programs Foundation of Ministry of Education of China (no. 20130162110043 awarded to Shuqiao Yao) and the Research Innovation Project for Postgraduate of Hunan Province, China (no. CX2011B068 awarded to Xiaocui Zhang). The authors are also very grateful to all volunteers who were willing to participate in this study and for their compliance to the study protocol without receiving any financial incentive.

\section{References}

[1] J. C. Shih and K. Chen, "MAO-A and -B gene knock-out mice exhibit distinctly different behavior," Neurobiology, vol. 7, no. 2, pp. 235-246, 1999.

[2] S. Z. Sabol, S. Hu, and D. Hamer, "A functional polymorphism in the monoamine oxidase A gene promoter," Human Genetics, vol. 103, no. 3, pp. 273-279, 1998.

[3] R. M. Denney, H. Koch, and I. W. Craig, "Association between monoamine oxidase A activity in human male skin fibroblasts and genotype of the MAOA promoter-associated variable number tandem repeat," Human Genetics, vol. 105, no. 6, pp. 542-551, 1999.

[4] S. B. Manuck, J. D. Flory, R. E. Ferrell, J. J. Mann, and M. F. Muldoon, "A regulatory polymorphism of the monoamine oxidase-A gene may be associated with variability in aggression, impulsivity, and central nervous system serotonergic responsivity," Psychiatry Research, vol. 95, no. 1, pp. 9-23, 2000.

[5] A. Caspi, J. McCray, T. E. Moffitt et al., "Role of genotype in the cycle of violence in maltreated children," Science, vol. 297, no. 5582, pp. 851-854, 2002.

[6] J. Samochowiec, K.-P. Lesch, M. Rottmann et al., "Association of a regulatory polymorphism in the promoter region of the monoamine oxidase A gene with antisocial alcoholism," Psychiatry Research, vol. 86, no. 1, pp. 67-72, 1999.

[7] Y. Y. Huang, S. P. Cate, C. Battistuzzi, M. A. Oquendo, D. Brent, and J. J. Mann, "An association between a functional polymorphism in the monoamine oxidase A gene promoter, impulsive traits and early abuse experiences," Neuropsychopharmacology, vol. 29, no. 8, pp. 1498-1505, 2004.

[8] J. Kim-Cohen, A. Caspi, A. Taylor et al., "MAOA, maltreatment, and gene-environment interaction predicting children's mental health: new evidence and a meta-analysis," Molecular Psychiatry, vol. 11, no. 10, pp. 903-913, 2006.

[9] D. L. Foley, L. J. Eaves, B. Wormley et al., "Childhood adversity, monoamine oxidase A genotype, and risk for conduct disorder," Archives of General Psychiatry, vol. 61, no. 7, pp. 738-744, 2004.

[10] K. W. Nilsson, R. L. Sjöberg, M. Damberg et al., "Role of monoamine oxidase A genotype and psychosocial factors in male adolescent criminal activity," Biological Psychiatry, vol. 59, no. 2, pp. 121-127, 2006.

[11] C. S. Widom and L. M. Brzustowicz, "MAOA and the "cycle of violence:" childhood abuse and neglect, MAOA genotype, and risk for violent and antisocial behavior," Biological Psychiatry, vol. 60, no. 7, pp. 684-689, 2006.

[12] S. R. H. Beach, G. H. Brody, T. D. Gunter, H. Packer, P. Wernett, and R. A. Philibert, "Child maltreatment moderates the association of MAOA with symptoms of depression and antisocial personality disorder," Journal of Family Psychology, vol. 24, no. 1, pp. 12-20, 2010.

[13] G. Guo, X.-M. Ou, M. Roettger, and J. C. Shih, "The VNTR 2 repeat in MAOA and delinquent behavior in adolescence and young adulthood: associations and MAOA promoter activity," European Journal of Human Genetics, vol. 16, no. 5, pp. 626-634, 2008.

[14] O. Cases, I. Self, J. Grimsby et al., "Aggressive behavior and altered amounts of brain serotonin and norepinephrine in mice lacking MAOA," Science, vol. 268, no. 5218, pp. 1763-1766, 1995. 
[15] T. K. Newman, Y. V. Syagailo, C. S. Barr et al., "Monoamine oxidase a gene promoter variation and rearing experience influences aggressive behavior in rhesus monkeys," Biological Psychiatry, vol. 57, no. 2, pp. 167-172, 2005.

[16] J. M. Mejia, F. R. Ervin, G. B. Baker, and R. M. Palmour, "Monoamine oxidase inhibition during brain development induces pathological aggressive behavior in mice," Biological Psychiatry, vol. 52, no. 8, pp. 811-821, 2002.

[17] J. Fan, J. Fossella, T. Sommer, Y. Wu, and M. I. Posner, "Mapping the genetic variation of executive attention onto brain activity," Proceedings of the National Academy of Sciences of the United States of America, vol. 100, no. 12, pp. 7406-7411, 2003.

[18] L. Passamonti, F. Fera, A. Magariello et al., "Monoamine oxidase-A genetic variations influence brain activity associated with inhibitory control: new insight into the neural correlates of impulsivity," Biological Psychiatry, vol. 59, no. 4, pp. 334-340, 2006.

[19] A. Cerasa, M. C. Gioia, F. Fera et al., "Ventro-lateral prefrontal activity during working memory is modulated by MAO A genetic variation," Brain Research, vol. 1201, pp. 114-121, 2008.

[20] A. Meyer-Lindenberg, J. W. Buckholtz, B. Kolachana et al., "Neural mechanisms of genetic risk for impulsivity and violence in humans," Proceedings of the National Academy of Sciences of the United States of America, vol. 103, no. 16, pp. 6269-6274, 2006.

[21] A. Cerasa, M. C. Gioia, A. Labate et al., "MAO A VNTR polymorphism and variation in human morphology: a VBM study," NeuroReport, vol. 19, no. 11, pp. 1107-1110, 2008.

[22] A. Cerasa, A. Quattrone, M. C. Gioia et al., "MAO A VNTR polymorphism and amygdala volume in healthy subjects," Psychiatry Research-Neuroimaging, vol. 191, no. 2, pp. 87-91, 2011.

[23] N. Alia-Klein, A. Kriplani, K. Pradhan et al., "The MAOA genotype does not modulate resting brain metabolism in adults," Psychiatry Research-Neuroimaging, vol. 164, no. 1, pp. 73-76, 2008.

[24] B. Biswal, F. Z. Yetkin, V. M. Haughton, and J. S. Hyde, "Functional connectivity in the motor cortex of resting human brain using echo-planar MRI," Magnetic Resonance in Medicine, vol. 34, no. 4, pp. 537-541, 1995.

[25] Y. F. Zang, Y. He, C. Z. Zhu et al., "Altered baseline brain activity in children with ADHD revealed by resting-state functional MRI," Brain and Development, vol. 29, pp. 83-91, 2007.

[26] A. Shmuel and D. A. Leopold, "Neuronal correlates of spontaneous fluctuations in fMRI signals in monkey visual cortex: mplications for functional connectivity at rest," Human Brain Mapping, vol. 29, no. 7, pp. 751-761, 2008.

[27] S. I. Gonçalves, J. C. de Munck, P. J. W. Pouwels et al., "Correlating the alpha rhythm to BOLD using simultaneous EEG/fMRI: inter-subject variability," NeuroImage, vol. 30, no. 1, pp. 203-213, 2006.

[28] Z. Zhang, G. Lu, Y. Zhong et al., "fMRI study of mesial temporal lobe epilepsy using amplitude of low-frequency fluctuation analysis," Human Brain Mapping, vol. 31, no. 12, pp. 1851-1861, 2010.

[29] M. J. Hoptman, X. N. Zuo, P. D. Butler et al., "Amplitude of lowfrequency oscillations in schizophrenia: a resting state fMRI study," Schizophrenia Research, vol. 117, no. 1, pp. 13-20, 2010.

[30] X. Q. Huang, S. Lui, W. Deng et al., "Localization of cerebral functional deficits in treatment-naive, first-episode schizophrenia using resting-state fMRI," NeuroImage, vol. 49, no. 4, pp. 2901-2906, 2010.
[31] Q. Jiao, J. Ding, G. Lu et al., "Increased activity imbalance in fronto-subcortical circuits in adolescents with major depression," PLoS ONE, vol. 6, no. 9, Article ID e25159, 2011.

[32] X. C. Zhang, X. L. Zhu, X. Wang et al., "First-episode medication-naive major depressive disorder is associated with altered resting brain function in the affective network," PLoS ONE, vol. 9, Article ID e85241, 2014.

[33] G. H. Jiang, Y. W. Qiu, X. L. Zhang et al., "Amplitude lowfrequency oscillation abnormalities in the heroin users: a resting state fMRI study," NeuroImage, vol. 57, no. 1, pp. 149-154, 2011.

[34] Y. Yin, L. Li, C. Jin et al., "Abnormal baseline brain activity in posttraumatic stress disorder: a resting-state functional magnetic resonance imaging study," Neuroscience Letters, vol. 498, no. 3, pp. 185-189, 2011.

[35] M. J. Lowe, M. D. Phillips, J. T. Lurito, D. Mattson, M. Dzemidzic, and V. P. Mathews, "Multiple sclerosis: lowfrequency temporal blood oxygen level-dependent fluctuations indicate reduced functional connectivity-initial results," Radiology, vol. 224, no. 1, pp. 184-192, 2002.

[36] L. Carrel, A. A. Cottle, K. C. Goglin, and H. F. Willard, "A firstgeneration X-inactivation profile of the human X chromosome," Proceedings of the National Academy of Sciences of the United States of America, vol. 96, no. 25, pp. 14440-14444, 1999.

[37] A. Fossati, A. Di Ceglie, E. Acquarini, and E. S. Barratt, "Psychometric properties of an Italian version of the Barrat Impulsiveness Scale-11 (BIS-11) in nonclinical subjects," Journal of Clinical Psychology, vol. 57, no. 6, pp. 815-828, 2001.

[38] K. J. Friston, S. Williams, R. Howard, R. S. J. Frackowiak, and R. Turner, "Movement-related effects in fMRI time-series," Magnetic Resonance in Medicine, vol. 35, no. 3, pp. 346-355, 1996.

[39] R. B. Lu, J. F. Lee, H. C. Ko et al., "No association of the MAO-Agene with alcoholism among Han Chinese males in Taiwan," Progress in Neuro-Psychopharmacology and Biological Psychiatry, vol. 26, pp. 457-461, 2002.

[40] S. Y. Huang, W. W. Lin, F. J. Wan et al., "Monoamine oxidaseA polymorphisms might modify the association between the dopamine D2 receptor gene and alcohol dependence," Journal of Psychiatry and Neuroscience, vol. 32, no. 3, pp. 185-192, 2007.

[41] M. B. Carpenter, "Gross anatomy of the brain," in Core Text of Neuroanatomy, pp. 31-43, Williams \& Wilkins, Baltimore, Md, USA, 2nd edition, 1978.

[42] W. J. Larsen, "Development of the brain and cranial nerves," in Essentials of Human Embryology, M. Strauss and D. Terry, Eds., pp. 273-302, Churchill Livingstone, New York, NY, USA, 2nd edition, 1998.

[43] J. W. Jahng, T. A. Houpt, T. C. Wessel et al., "Localization of monoamine oxidase $\mathrm{A}$ and $\mathrm{B}$ mRNA in the rat brain by in situ hybridization," Synapse, vol. 25, pp. 30-36, 1997.

[44] T. E. Moffitt, G. L. Brammer, A. Caspi et al., "Whole bloodserotonin relates to violence in an epidemiological study," Biological Psychiatry, vol. 43, no. 6, pp. 446-457, 1998.

[45] S. Howell, G. Westergaard, B. Hoos et al., "Serotonergic influences on life-history outcomes in free-ranging male rhesus macaques," The American Journal of Primatology, vol. 69, no. 8, pp. 851-865, 2007. 


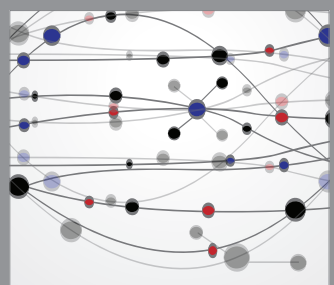

The Scientific World Journal
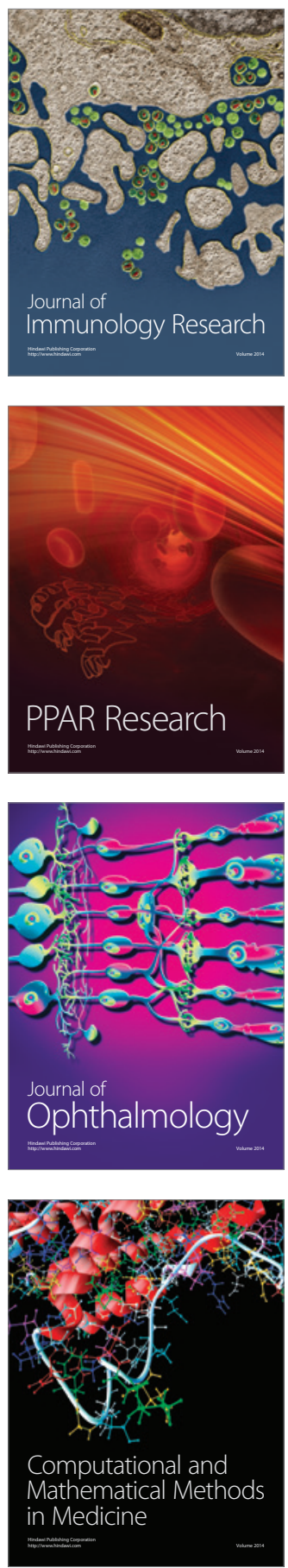

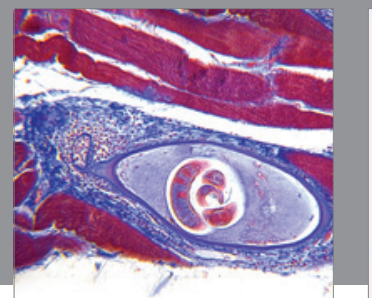

Gastroenterology

Research and Practice
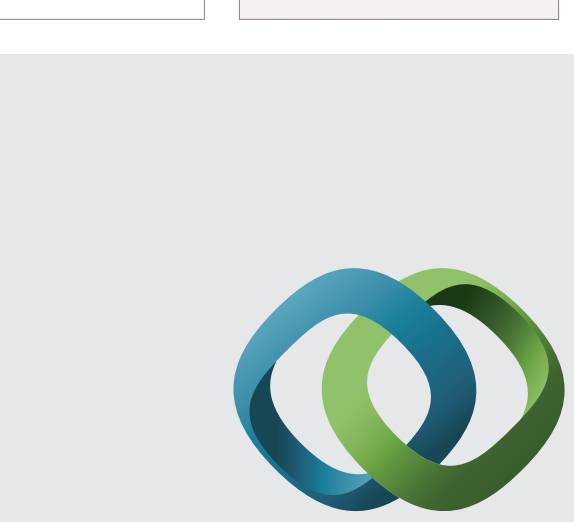

\section{Hindawi}

Submit your manuscripts at

http://www.hindawi.com
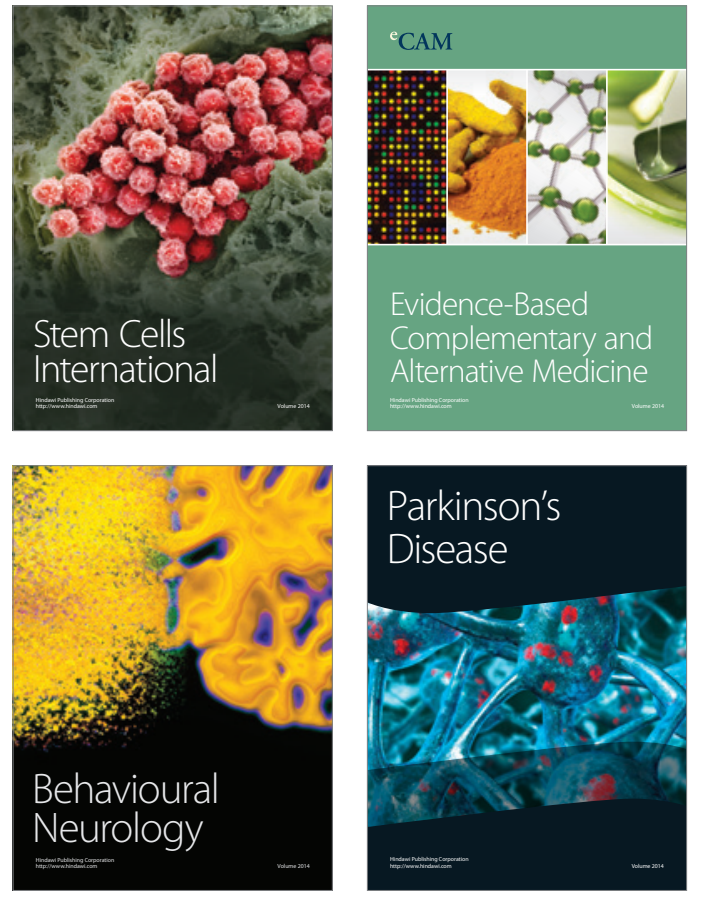
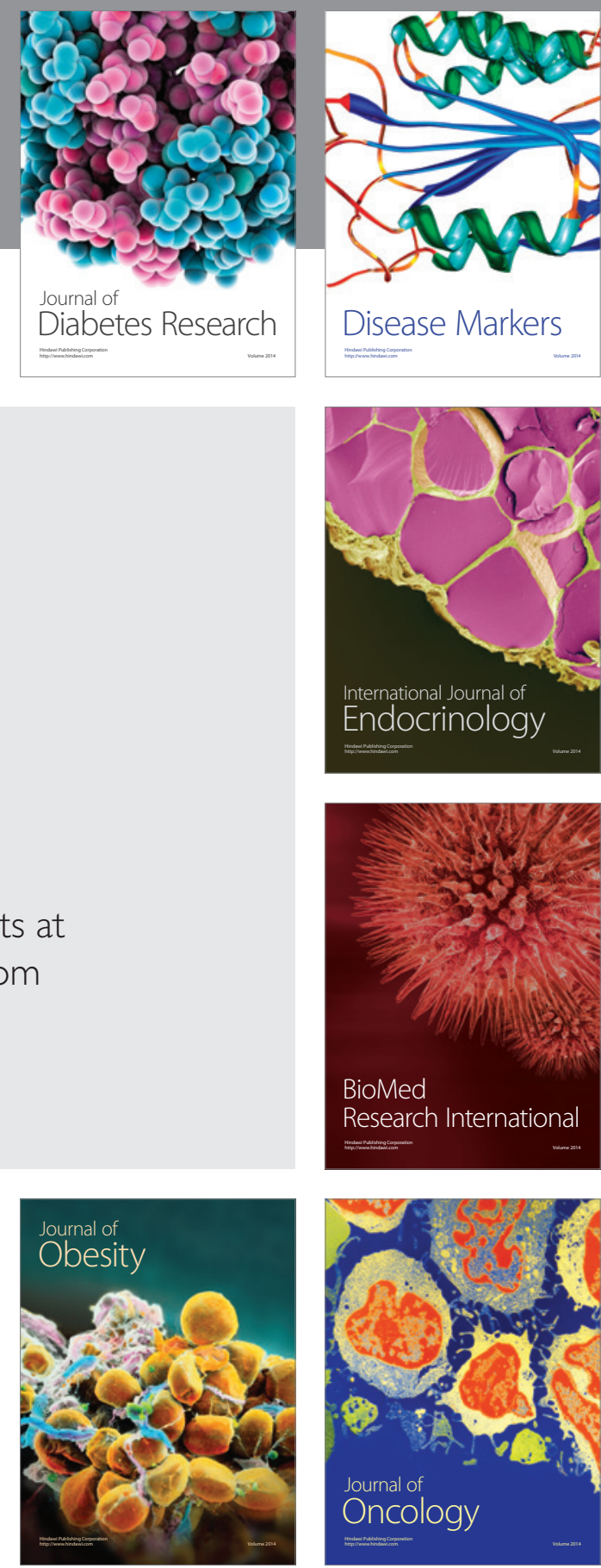

Disease Markers
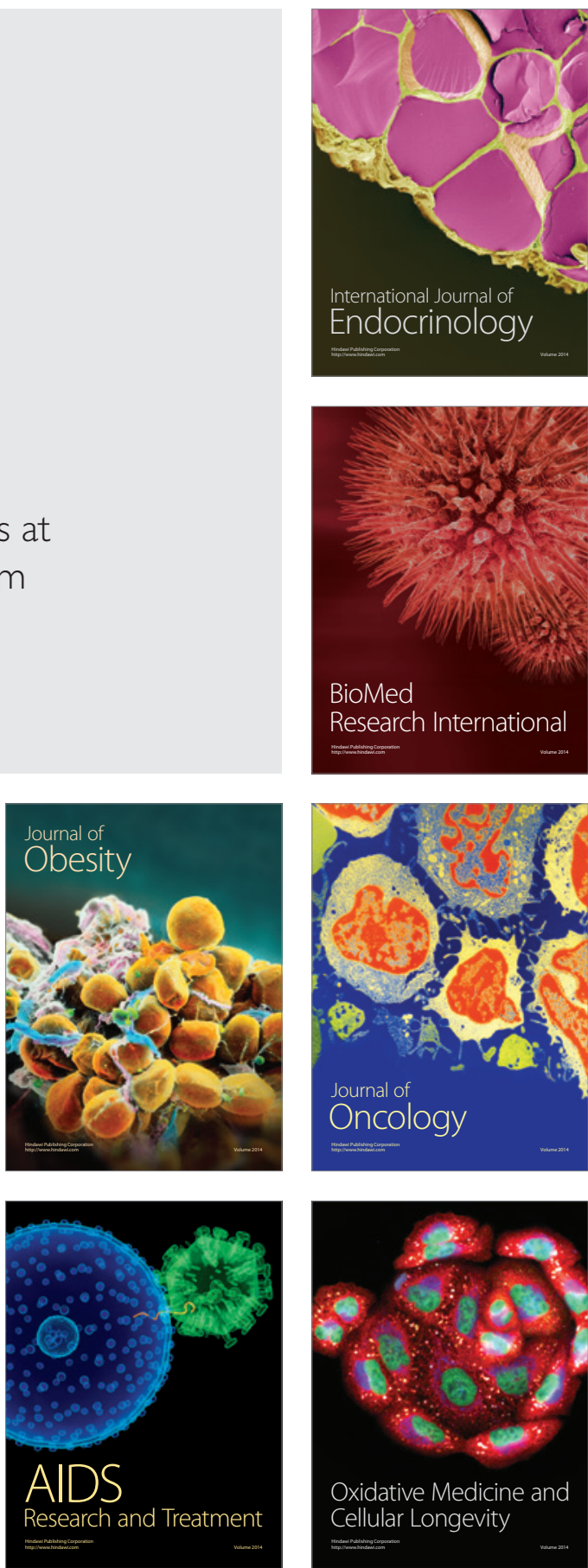